\title{
Acute eosinophilic pneumonia triggered by secondhand cigarette smoke exposure in an elderly man
}

\author{
Montserrat Diaz-Abad ${ }^{1}$, Laura Malone ${ }^{2}$, Liaqat Ali ${ }^{3}$, Edward M. Pickering ${ }^{1}$, Ashutosh Sachdeva ${ }^{1}$ \\ ${ }^{1}$ Department of Medicine, University of Maryland School of Medicine, Baltimore, MD; ${ }^{2}$ Department of Pathology, \\ University of Maryland Medical Center, Baltimore, MD; ${ }^{3}$ Department of Medicine, University of Maryland Medical \\ Center Midtown Campus, Baltimore, MD, USA
}

\begin{abstract}
The spectrum of eosinophilic lung diseases comprises a diverse group of pulmonary disorders associated with tissue or peripheral eosinophilia. Acute eosinophilic pneumonia (AEP) is an uncommon eosinophilic lung disease that can be idiopathic, but identifiable causes include medications, inhalational exposures and infections. Most cases in the literature are associated with first-time cigarette smoking or resuming smoking. Herein, we present a case of AEP in an elderly man triggered by exposure to secondhand tobacco smoke, in whom a transbronchial biopsy was diagnostic. The patient recovered fully with glucocorticoid therapy without recurrence after avoiding further secondhand smoke.
\end{abstract}

Correspondence: Montserrat Diaz-Abad, Division of Pulmonary and Critical Care Medicine, University of Maryland School of Medicine, 100 North Greene Street, Room 204, Baltimore MD 21201, USA. Tel. +1.410.706.4771 - Fax: +1.410.706.0345.

E-mail:mdiaz@som.umaryland.edu

Key words: Acute eosinophilic pneumonia; secondhand smoking; bronchoalveolar lavage; elderly, diagnosis.

Contributions: MDA, manuscript writing; LM, LA, EMP, AS, review, inclusion of data according to single specialties and contribution to the case(s). All authors reviewed the manuscript and approved it.

Conflict of interest: MDA, LM, LA, EMP declare no conflict of interest. AS reports grants, personal fees and non-financial support from Medtronic, grants and non-financial support from Olympus, personal fees from CSA Medical, personal fees from Pinnacle Biologics, outside the submitted work.

Funding: No funding was secured for this study.

Received for publication: 1 August 2018.

Accepted for publication: 25 January 2019.

(C) Copyright M. Diaz-Abad et al., 2019

Licensee PAGEPress, Italy

Monaldi Archives for Chest Disease 2019; 89:985

doi: 10.4081/monaldi.2019.985

This article is distributed under the terms of the Creative Commons Attribution Noncommercial License (by-nc 4.0) which permits any noncommercial use, distribution, and reproduction in any medium, provided the original author(s) and source are credited.

\section{Introduction}

Acute eosinophilic pneumonia (AEP) is an uncommon eosinophilic lung disease. Diagnosis is based on an acute onset of symptoms, lung opacities on chest imaging and pulmonary eosinophilia. Commonly confused with community-acquired pneumonia, this condition improves within days with corticosteroid treatment. While some cases are idiopathic, multiple causes have been identified, most importantly tobacco smoking, along with other toxins, medications and infection [1]. Exposure to secondhand smoke has been reported in only two cases [2,3].

We present a case of an elderly patient with AEP triggered by exposure to secondhand tobacco smoke diagnosed by transbronchial biopsy.

\section{Case Report}

A 72-year-old man experienced 2 weeks of progressive dyspnea with exertion, 1 week of malaise, fatigue, palpitations, cough productive of clear sputum, and 3 days of fever. He reported dyspnea with minimal activity on admission. He was generally healthy at baseline, and had quit cigarette smoking 25 years ago (15 pack-year history). He occasionally smoked marijuana without any recent change in pattern or intensity. He reported very bothersome and recent exposure to heavy secondhand tobacco smoke in enclosed rooms at home after starting a new relationship with someone who smoked cigarettes heavily.

On admission, temperature was $38.3 \mathrm{C}$ and white blood cell count (WBC) was $24.4 \mathrm{~K} / \mathrm{mcL}$ cell differential: eosinophils $20.1 \%$ (4.9 K/mcL), neutrophils $41.8 \%$, lymphocytes $32.7 \%$, monocytes $5.2 \%$ and basophils $0.2 \%$. $\mathrm{SpO}_{2}$ was $92 \%$; nasal cannula (NC) oxygen $2 \mathrm{~L} / \mathrm{min}$ was started. Scattered rhonchi, decreased breath sounds at bases, crackles in mid lungs, normal respiratory effort and a systolic murmur grade $3 / 6$ in the right upper sternal border were noted. Chest x-ray showed bibasilar patchy airspace opacities, and computed tomography showed areas of consolidation in all lobes most evident in the lower lobes, bronchovascular bundle thickening, septal markings, enlarged hilar and subcarinal lymph nodes and a small right pleural effusion (Figure 1). The findings were most compatible with pneumonia, but sarcoidosis or lymphoma were in the differential.

The patient was started on ceftazidime and vancomycin for possible community acquired pneumonia and sepsis. Blood and sputum cultures, respiratory viral panel, Legionella and Streptococcus pneumoniae urinary antigens were all negative. 
Because of the peripheral eosinophilia, eosinophilic lung disease, in particular AEP was also suspected. Immunoglobulin (Ig)E, antineutrophil cytoplasmic antibody panel, C-reactive protein and erythrocyte sedimentation rate were normal except for total $\mathrm{IgE}$ elevated at $484 \mathrm{IU} / \mathrm{ml}$. Echocardiogram demonstrated severe calcific aortic stenosis with valve area $0.8 \mathrm{~cm}^{2}$ and moderate pulmonary hypertension; bronchoscopy was postponed pending cardiac evaluation. Over the next 2 days there was marked clinical deterioration: WBC peaked at $48.8 \mathrm{~K} / \mathrm{mcL}$, with worsening bibasilar opacification and new bilateral pleural effusions on chest x-ray, increased work of breathing and tachypnea. Arterial blood gas showed severe hypoxemia: $\mathrm{pH} 7.36, \mathrm{PCO}_{2} 42, \mathrm{PO}_{2} 62, \mathrm{SaO}_{2} 88$ on $5 \mathrm{l} / \mathrm{min}$ oxygen NC. Antibiotics were switched to linezolid and piperacillin-tazobactam, and due to imminent respiratory failure methylprednisolone $60 \mathrm{mg}$ intravenously every $12 \mathrm{~h}$ was started. Ultrasound revealed a moderate right and large left pleural effusion, with $850 \mathrm{ml}$ serosanguineous fluid removed via a left thoracentesis. Because of prior good functional status, the patient was deemed stable from a cardiac standpoint for bronchoscopy.

Two days later, WBC was $39.8 \mathrm{~K} / \mathrm{mcl}$ with normalization of eosinophil count to $0.4 \%(0.2 \mathrm{~K} / \mathrm{mcl})$. The patient felt much better on $3 \mathrm{l} / \mathrm{min}$ oxygen NC. Diagnostic bronchoscopy was performed while intubated. Diffuse tracheobronchomalacia with airway collapse was noted despite positive end-expiratory pressure $8 \mathrm{~cm}$ $\mathrm{H}_{2} \mathrm{O}$. Bronchoalveolar lavage (BAL) was performed in the lingula and left upper lobe with poor return in both sites. Transbronchial needle aspiration with endobronchial ultrasound of the enlarged lymph nodes and 6 transbronchial biopsies of the lung parenchyma were also performed without complications.

Over the following 2 days, WBC continued to decrease, oxygen was weaned off, and symptoms resolved with improved bibasilar opacities on chest X-ray. BAL was acellular and non-diagnostic. Lymph node cytology was normal. Pathology revealed eosinophilic pneumonia (Figure 2), confirming the diagnosis of AEP. The patient was discharged on prednisone $40 \mathrm{mg}$ daily with follow up in 4 weeks to initiate taper. He came off prednisone and avoided further exposure to secondhand smoke. One year later there was no recurrence (Figure 1).

\section{Discussion}

AEP is an uncommon eosinophilic lung disease. We present a case of an elderly patient with AEP triggered by exposure to secondhand tobacco smoke, confirmed by transbronchial biopsy. This patient developed AEP attributed to recent heavy indoor secondhand smoke exposure. There have been only two previously reported cases of secondhand smoke associated with AEP $[2,3]$. Other potential triggers were investigated. Although the patient occasionally smoked marijuana and this has been also associated with AEP in two cases [4,5], there had been no change in intensity or frequency of use. At 72 years old, the patient was also significantly older than the usual reported age of 20 to 40 years of age [1].

AEP is frequently confused with community-acquired pneumonia upon presentation. It is very important to make the correct diagnosis as this disease responds completely to corticosteroid treatment but can rapidly lead to respiratory failure and be fatal if misdiagnosed. The hallmark of AEP is pulmonary eosinophilia, with BAL $>25 \%$ eosinophils in cell differential or eosinophilic pneumonia on lung biopsy required for diagnosis. Peripheral blood eosinophilia may not be present at presentation but eventually occurs in most cases [1]. In this case, both the recent inhalational exposure and peripheral eosinophilia were early clues to a possible AEP diagnosis.
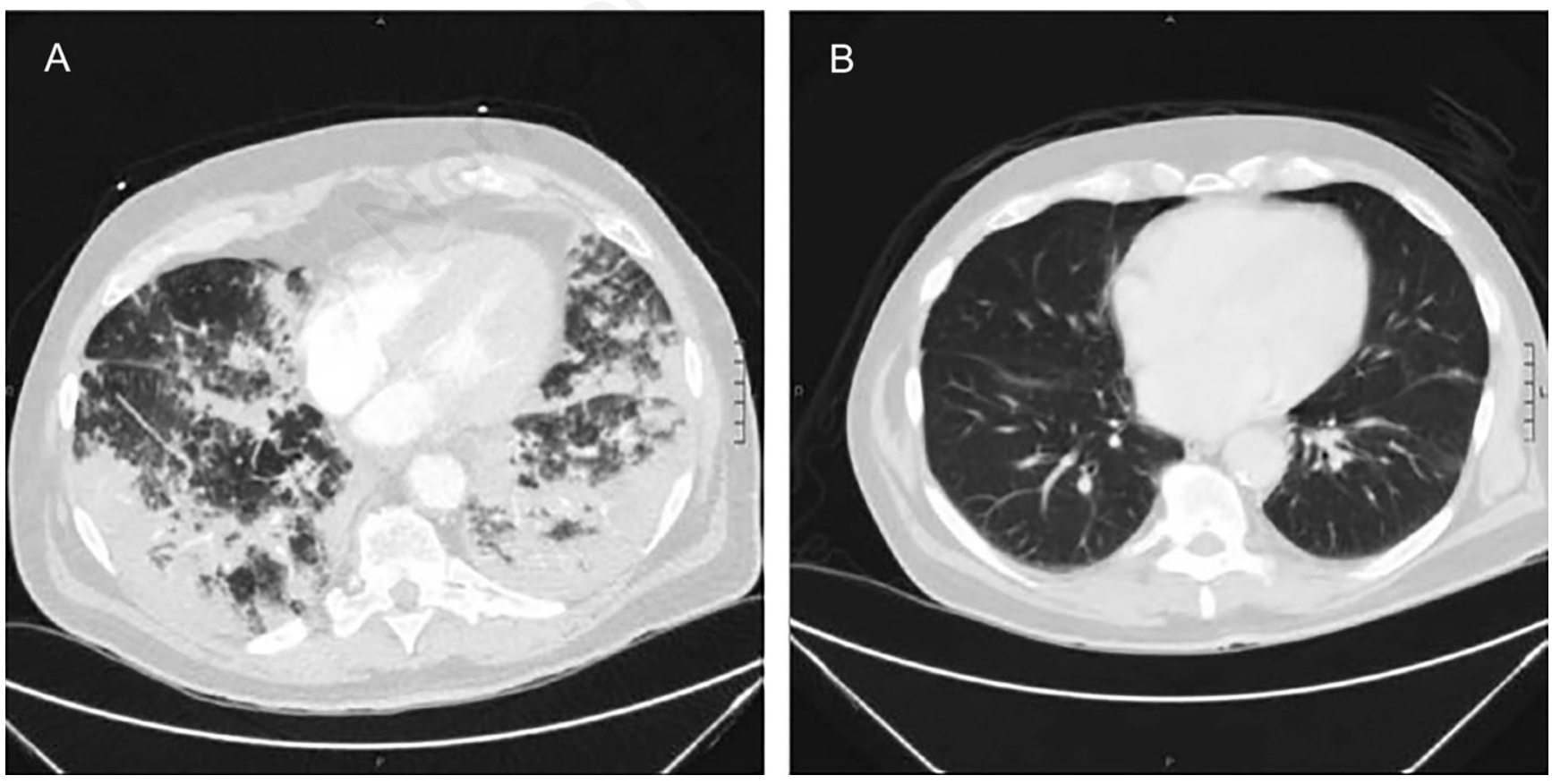

Figure 1. A) Computed tomography of the chest showing areas of dense parenchymal consolidation in the lower lobes, thickening of the bronchovascular bundle and septal markings. B) Five months later, findings revolved. 
Lung biopsy -usually transbronchial- is less likely to be performed in AEP because the diagnosis can be made with BAL and patients may get ill quickly making biopsy riskier, but may be indicated when there are atypical features that suggest an alternative diagnosis [1]. In this case, imaging suggested the possibility of sarcoidosis or lymphoma, and thus the decision to perform biopsy, without which the diagnosis would have been missed. Given paucity of literature, it is difficult to provide a concrete number of passes required for achieving diagnosis of AEP. We recommend obtaining 4-8 transbronchial biopsies/pieces (with 4 adequate pieces) when evaluating patients with diffuse parenchymal opacities. We performed a total of 6 biopsies, which is similar to the number $(6 \pm 2)$ performed in a study of 169 immunocompetent outpatients with various pulmonary diagnoses [6] and represents common practice amongst various pulmonologists.

There are potential pitfalls associated with relying only on BAL to make a diagnosis and start treatment for AEP. We favor that if a good quality BAL can be obtained resulting in a reliable cell count assessment, and clinical-radiological correlation favors AEP, a lower cut-off value of eosinophil count could be used as diagnostic criteria; but which cut-off value to use remains unclear. However, a high quality BAL $(>50 \%$ return from representative lung segment, with visible surfactant) resulting in a reliable cell count may not be possible due to patient (airway collapsibility, cough interfering with wedge position of the scope, etc.) or operator factors, and in these situations transbronchial biopsies may be helpful provided the patient has no contraindications for the sampling and the procedure is feasible. BAL eosinophilia has also been shown to resolve a few days after the start of corticosteroid therapy [7]. Additionally, BAL eosinophils can be degranulated and demonstrate multiple nuclear lobes, resembling neutrophils, and this could potentially lead to a mistaken neutrophilic pattern on BAL [1]. In our case transbronchial biopsy was quite helpful in making a diagnosis.

While $>25 \%$ BAL eosinophils is the diagnostic criteria if no biopsy, this level might also be too stringent. There have been several cases of AEP diagnosed with a lower eosinophil level or even a normal count. There is a case report of a BAL with neutrophil predominance without eosinophils [8]. The diagnosis was confirmed with transbronchial biopsy and the authors speculated this may have been related to early disease. In a series of 22 patients with AEP, 3 patients (14\%) also had a normal BAL eosinophil count $(<1 \%)$ [9]. Other series have also reported values $<25 \%$. In 45 patients with AEP, only $36(80 \%)$ had BAL eosinophils $>25 \%$ [10]. A case series of AEP reported [11] $>25 \%$ eosinophils in BAL in 2 out of 6 cases, and in only 16 out of $31(52 \%)$ cases in its accompanying literature review. In another report involving 2 cases, one had BAL eosinophils of $17 \%$ [12]; the authors discussed the difficulty with the current diagnostic criteria $[6,13]$, and argued that adherence to strict cut-offs may limit the number of diagnoses. They proposed substituting $>25 \%$ BAL eosinophils with any abnormal value of BAL \% eosinophils [12].

\section{Conclusions}

To our knowledge, the current case is the third report of a patient with AEP associated with secondhand tobacco smoke exposure. In addition, this was an elderly patient and the diagnosis was confirmed by transbronchial biopsy because of a nondiagnostic BAL. Bronchoscopy was delayed prompting the start of empiric corticosteroid therapy for impending respiratory failure with almost immediate clinical improvement. This illustrates the potential role for empiric therapy in suspected AEP after a careful risk/benefit assessment, in cases where diagnostic procedures are not feasible or nondiagnostic. This case and the review of the prior cases also bring attention to potential limitations of BAL testing to diagnose and, most importantly, initiate therapy in this disease, and suggest that current BAL diagnostic criteria for AEP may need to be reevaluated. The question of whether a specific cut-off value of BAL \% eosinophils in an adequate sample would be diagnostic, or whether the addition of transbronchial biopsy may be required needs further study.

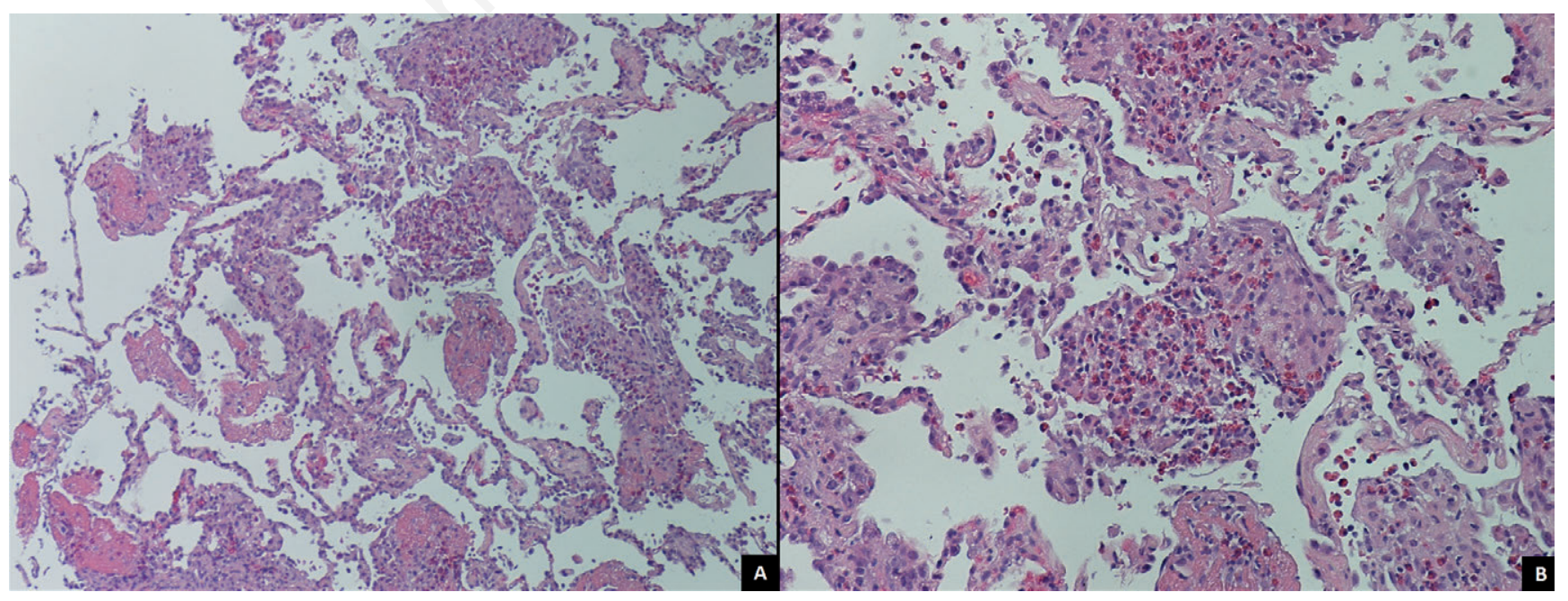

Figure 2. A) Low power view of the lung showing intra-alveolar eosinophils mixed with macrophages and fibrin; there is also interstitial eosinophilia with perivascular predilection; the bronchiole is spared showing no significant inflammation or eosinophilia; H\&E, $\mathrm{x} 100$. B) Higher power view of the lung showing abundant interstitial and intra-alveolar eosinophils associated with reactive pneumocytes; H\&E, x200. 


\section{References}

1. De Giacomi F, Vassallo R, Yi ES, Ryu JH. Acute Eosinophilic pneumonia: causes, diagnosis and management. Am J Respir Crit Care Med 2018;197:728-36.

2. Komiya K, Teramoto S, Kawashima M, et al. A case of acute eosinophilic pneumonia following short-term passive smoking: an evidence of very high level of urinary cotinine. Allergol Int 2010;59:421-3.

3. Chung MK, Lee SJ, Kim MY, et al. Acute eosinophilic pneumonia following secondhand cigarette smoke exposure. Tuberc Respir Dis (Seoul) 2014;76:188-91.

4. Liebling PD, Siu S. A novel cause of eosinophilic pneumonia: recreational marijuana exposure. J Bronchology Interv Pulmonol 2013;20:183-5.

5. Natarajan A, Shah P, Mirrakhimov AE, Hussain N. Eosinophilic pneumonia associated with concomitant cigarette and marijuana smoking. BMJ Case Rep 2013;2013: pii:bcr 2013009001.

6. Hernández Blasco L, Sánchez Hernández IM, Villena Garrido $\mathrm{V}$, et al. Safety of the transbronchial biopsy in outpatients. Chest 1991;99:562-5.
7. Philit F, Etienne-Mastroïanni B, Parrot A, et al. Idiopathic acute eosinophilic pneumonia: a study of 22 patients. Am J Respir Crit Care Med 2002;166:1235-9.

8. Iwami $\mathrm{T}$, Umemoto $\mathrm{S}$, Ikeda $\mathrm{K}$, et al. A case of acute eosinophilic pneumonia. Evidence for hypersensitivity-like pulmonary reaction. Chest 1996;110:1618-21.

9. De Giacomi F, Decker PA, Vassallo R, Ryu JH. Acute eosinophilic pneumonia: correlation of clinical characteristics with underlying cause. Chest 2017;152:379-385.

10. Bartal C, Sagy I, Barski L. Drug-induced eosinophilic pneumonia: A review of 196 case reports. Medicine (Baltimore) 2018;97:e9688.

11. Hirai J, Hagihara M, Haranaga S, et al. Eosinophilic pneumonia caused by daptomycin: Six cases from two institutions and a review of the literature. J Infect Chemother 2017;23: 245-249.

12. Phillips J, Cardile AP, Patterson TF, Lewis JS 2nd. Daptomycin-induced acute eosinophilic pneumonia: analysis of the current data and illustrative case reports. Scand J Infect Dis 2013;45:804-8.

13. Kebbe J, Abdo T. Interstitial lung disease: the diagnostic role of bronchoscopy. J Thorac Dis 2017;9:S996-S1010. 